\title{
Research Article \\ Spectrum of a Differential Operator with Periodic Generalized Potential
}

\author{
Mehmet Sahin and Manaf Dzh. Manafov
}

Received 2 April 2007; Revised 11 July 2007; Accepted 5 October 2007

Recommended by Agacik Zafer

We study some spectral problems for a second-order differential operator with periodic potential. Notice that the given potential is a sum of zero- and first-order generalized functions. It is shown that the spectrum of the investigated operator consists of infinite number of gaps whose length limit unlike the classic case tends to nonzero constant in some place and to infinity in other place.

Copyright $\odot 2007$ M. Sahin and M. Dzh. Manafov. This is an open access article distributed under the Creative Commons Attribution License, which permits unrestricted use, distribution, and reproduction in any medium, provided the original work is properly cited.

\section{Introduction}

The problem of studying spectral characteristics of the differential equation

$$
\ell_{\alpha \beta}[y] \equiv-y^{\prime \prime}+q(x) y+\left(\alpha \sum_{n=-\infty}^{m} \delta(x-n)+\beta \sum_{n=m+1}^{\infty} \delta^{\prime}(x-n)\right) y=\lambda y, \quad-\infty<x<\infty
$$

arises in many model problems of quantum mechanics. In this formula, $q(x)$ is a real periodic locally square integrable function $(q(x+1)=q(x)), \delta(x)$ is Dirac's function, and $\delta^{\prime}(x)$ is its derivative function, $\alpha, \beta \neq 0$ are real numbers.

Since in the formula (1.1) there is a series composed of $\delta$ and $\delta^{\prime}$ functions, it is necessary to indicate mathematically correct description of this formula. For this purpose, following the paper [1], we include the problem (1.1) into the general scheme of the theory of self-adjoint extensions of symmetric operators in Hilbert space (see [2]).

We denote by $D_{0}^{\infty}$ a totality of finite infinitely differentiable functions $y(x)$ on the whole of the axis, such that $y(n)=0(n=m, m-1, m-2, \ldots)$ and $y^{\prime}(n)$ $=0(n=m+1, m+2, \ldots)$. On the set $D_{0}^{\infty}$, by means of the differential expression 
$\ell_{00}[y] \equiv-y^{\prime \prime}+q(x) y$, we define the operator $L_{00}(q)$ in the space $L_{2}(-\infty, \infty)$. The operator $L_{00}(q)$ is symmetric, nonclosed operator. The closure of the operator $L_{00}(q)$ in the metric $L_{2}(-\infty, \infty)$ is denoted by $L$. The operator $L$ has same deficiency number, and therefore, by Neumann theorem it admits self-adjoint extension $\tilde{L}$, where $L \subset \tilde{L} \subset L^{*}$ (see [3]). Notice that the definition domain $D\left(L^{*}\right)$ of the operator $L^{*}$ consists of all continuous functions $f(x) \in L_{2}(-\infty, \infty)(x \neq n=0, \pm 1, \pm 2, \ldots)$, for which $\ell_{00}[f] \in L_{2}(-\infty, \infty)$. On the functions from $D\left(L^{*}\right)$ for $x \neq n=0, \pm 1, \pm 2, \ldots$, the operator $L^{*}$ is given by the formula $L^{*} f=-f^{\prime \prime}+q(x) f$. On the functions from $D\left(L^{*}\right)$ subjected to the conditions

$$
\begin{gathered}
y(n)=y(n-0)=y(n+0), \quad y^{\prime}(n+0)-y^{\prime}(n-0)=\alpha y(n) \quad(n=m, m-1, \ldots), \\
y(n+0)-y(n-0)=\beta y^{\prime}(n), \quad y^{\prime}(n)=y^{\prime}(n-0)=y^{\prime}(n+0) \quad(n=m+1, m+2, \ldots)
\end{gathered}
$$

by means of differential expression $\ell_{00}[y]$, we define the operator $L_{\alpha \beta}(q)$. From results of $[4,5]$, it holds the following.

Theorem 1.1. The operator $L_{\alpha \beta}(q)$ is a self-adjoint extension of the operator $L$ in $L_{2}(-\infty$, $\infty)$.

Notice that the problem on studying spectral characteristics of (1.1) is equivalent to studying spectral properties of the operator $L_{\alpha \beta}(q)$ in the Hilbert space $L_{2}(-\infty, \infty)$ (see $[6,7])$.

We formulate the main results of the paper.

We denote by $\theta(x, \lambda)$ and $\varphi(x, \lambda)$ the solutions of the equation

$$
\ell_{00}[y] \equiv-y^{\prime \prime}+q(x) y=\lambda y
$$

defined by the following boundary conditions at $x=0$ :

$$
\theta(0, \lambda)=\varphi^{\prime}(0, \lambda)=1, \quad \theta^{\prime}(0, \lambda)=\varphi(0, \lambda)=0
$$

Let us consider the functions $F_{\alpha}(\lambda)=\theta(1, \lambda)+\varphi^{\prime}(1, \lambda)+\alpha \varphi(1, \lambda)$ and $F_{\beta}(\lambda)=\theta(1, \lambda)+$ $\varphi^{\prime}(1, \lambda)+\beta \theta^{\prime}(1, \lambda)$. In Section 2 it is proved that the functions $\Phi_{ \pm}^{\alpha}(\lambda)=F_{\alpha}(\lambda) \pm 2$ and $\Phi_{ \pm}^{\beta}(\lambda)=F_{\beta}(\lambda) \pm 2$ have only real zeros whose multiplicity does not exceed two. Denote by $k_{j}(j=0, \pm 1, \pm 2, \ldots)$, the roots of the equations $F_{\alpha}(\lambda)=2$ and $F_{\beta}(\lambda)=2$ enumerated in nondecreasing order, and by $\mu_{j}$ - the roots of the equations $F_{\alpha}(\lambda)=-2$ and $F_{\beta}(\lambda)=-2(j=0, \pm 1, \pm 2, \ldots)$. Besides, let $\sigma_{j}(j=m, m-1, m-2, \ldots)$ be the zeros of the functions $\varphi(1, \lambda)$ and $\sigma_{j}(j=m+1, m+2, \ldots)$ be the zeros of the function $\theta^{\prime}(1, \lambda)$.

In Sections 2 and 3, we prove the following main theorem.

Theorem 1.2. (a) The sequence of numbers $k_{j}, \mu_{j}$, and $\sigma_{j}$ satisfies the inequalities for $j>0$ :

$$
\cdots<k_{-j}<\mu_{-j} \leq \sigma_{-j+1} \leq \mu_{-j+1}<\cdots \leq k_{j} \leq \sigma_{j+1} \leq k_{j+1}<\mu_{j+1} \leq \cdots
$$


(b) the spectrum of the operator $L_{\alpha \beta}(q)$ is absolutely continuous set $S=S_{1} \cup S_{2}$ and negative eigenvalue that number is not exceeding two, where

$$
S_{1}=\bigcup_{j=-\infty}^{\infty}\left[k_{2 j}, \mu_{2 j}\right], \quad S_{2}=\bigcup_{j=-\infty}^{\infty}\left[\mu_{2 j+1}, k_{2 j+1}\right] .
$$

In sequel, the segments of the real axis $\left[k_{2 j}, \mu_{2 j}\right],\left[\mu_{2 j+1}, k_{2 j+1}\right](j=0, \pm 1, \pm 2, \ldots)$ will be said to be the bands of the spectrum of the operator $L_{\alpha}(q)$, and the intervals $\left[\mu_{2 j}, \mu_{2 j+1}\right]$, $\left[k_{2 j+1}, k_{2 j+2}\right](j=0, \pm 1, \pm 2, \ldots)$ will be called the gaps.

Alongside with the operator $L_{\alpha \beta}(q)$, we consider the operator $L_{00}(q)$. A is known (see $[8])$, the spectrum of this operator also consists of the segments of a real axis separated by gaps, and it is possible the case when the number of the spectrum zones is finite (e.g., if $q(x)=0$ ). But if the number of the zones is infinite, then the lengths of gaps in the spectrum tend to zero. However, for the operator $L_{\alpha \beta}(q)$, the number of spectrum zones is always infinite and the lengths of gaps tend to nonzero constant in some place and to infinity in other place. Notice that for $m=-\infty$, it is investigated in [9]. By $m= \pm \infty$, it is studied the asymptotic case of the bands of the spectrum and gaps in [10].

\section{Distribution of zeros of functions $\Phi_{ \pm}^{\alpha}(\lambda)$ and $\Phi_{ \pm}^{\beta}(\lambda)$}

By investigating the spectrum of the operator $L_{\alpha \beta}(q)$ formulated in introduction, we will need general properties of functions $\Phi_{ \pm}^{\alpha}(\lambda)$ and $\Phi_{ \pm}^{\beta}(\lambda)$. In sequel, for reduction of writing we will use the following notation:

$$
\varphi \equiv \varphi(1, \lambda) ; \quad \varphi^{\prime} \equiv \varphi^{\prime}(1, \lambda) ; \quad \theta \equiv \theta(1, \lambda) ; \quad \theta^{\prime} \equiv \theta^{\prime}(1, \lambda)
$$

It holds the following.

LEMma 2.1. The functions $\Phi_{ \pm}(\lambda)$ have only real zeros and these zeros form a denumerable set with concentration point $\lambda=+\infty$.

Proof. Let in the Hilbert space $L_{2}(0,1)$ consider the problem:

$$
\begin{gathered}
-y^{\prime \prime}+q(x) y=\lambda y, \quad 0<x<1, \\
y(0)=\mp y(1) ; \quad y^{\prime}(0)=\mp\left(y^{\prime}(1)+\alpha y(1)\right) .
\end{gathered}
$$

It is easy to check that this spectral problem is self-adjoint and its spectrum coincides with the zeros of the entire function $\Phi_{ \pm}^{\alpha}(\lambda)$. Whence, the existence of zeros of the function $\Phi_{ \pm}^{\alpha}(\lambda)$ follows.

Similarly, consider in the space $L_{2}(0,1)$ the self-adjoint spectral problem

$$
\begin{array}{cl}
-y^{\prime \prime}+q(x) y=\lambda y, & 0<x<1, \\
y(0)=\mp\left(y(1)+\beta y^{\prime}(1)\right), & y^{\prime}(0)=\mp y^{\prime}(1) .
\end{array}
$$

It is not hard to be convinced that its spectrum coincides, with zero of the entire function $\Phi_{ \pm}^{\beta}(\lambda)$. Whence, the existence of zeros follows. 
Since $\Phi_{ \pm}^{\alpha}(\lambda)$ and $\Phi_{ \pm}^{\beta}(\lambda)$ are fractional orders, then they have infinite number of zeros. Consequently, these zeros form a denumerable set with concentration points $\lambda=+\infty$.

Lemma 2.2. Let $\lambda$ be a real number. Assume that $F_{\alpha}(\lambda)=2, F_{\alpha}^{\prime}(\lambda)=0$. Then, at this value of $\lambda$,

$$
\theta=1, \quad \varphi=0, \quad \theta+\alpha \theta^{\prime}=0, \quad \varphi^{\prime}=1, \quad F_{\alpha}^{\prime \prime}(\lambda)<0 .
$$

Lemma 2.3. Let $\lambda$ be a real number. Assume that $F_{\alpha}(\lambda)=-2, F_{\alpha}^{\prime}(\lambda)=0$. Then, at this value of $\lambda$,

$$
\theta=-1, \quad \varphi=0, \quad \theta+\alpha \theta^{\prime}=0, \quad \varphi^{\prime}=-1, \quad F_{\alpha}^{\prime \prime}(\lambda)>0 .
$$

Lemma 2.4. Let $\lambda$ be a real number. Assume that $F_{\beta}(\lambda)=2, F_{\beta}^{\prime}(\lambda)=0$. Then, at this value of $\lambda$,

$$
\theta=1, \quad \theta^{\prime}=0, \quad \varphi+\beta \varphi^{\prime}=0, \quad \varphi^{\prime}=1, \quad F_{\beta}^{\prime \prime}(\lambda)<0 .
$$

Lemma 2.5. Let $\lambda$ be a real number. Assume that $F_{\beta}(\lambda)=-2, F_{\beta}^{\prime}(\lambda)=0$. Then, at this value of $\lambda$,

$$
\theta=-1, \quad \theta^{\prime}=0, \quad \varphi+\beta \varphi^{\prime}=0, \quad \varphi^{\prime}=-1, \quad F_{\beta}^{\prime \prime}(\lambda)>0
$$

Lemmas 2.2, 2.3, 2.4, and 2.5 are proved similarly. Therefore, we prove Lemma 2.5.

Proof. Assume that $\theta^{\prime} \neq 0$. Then, taking into account that $\theta \varphi^{\prime}-1=\theta^{\prime} \varphi$, using the formula for derivatives $\partial \theta(\xi, \lambda) / \partial \lambda, \partial \theta^{\prime}(\xi, \lambda) / \partial \lambda$, and $\partial \varphi^{\prime}(\xi, \lambda) / \partial \lambda$ (see [8, Part 2, page 351]), we find

$$
\begin{aligned}
\frac{d F_{\beta}(\lambda)}{d \lambda}= & \theta^{\prime} \int_{0}^{1}\left[\varphi(\xi, \lambda)+\frac{\theta+\beta \theta^{\prime}-\varphi^{\prime}}{2 \theta^{\prime}} \theta(\xi, \lambda)\right]^{2} d \xi \\
& +\frac{4-\left(\theta+\beta \theta^{\prime}-\varphi^{\prime}\right)^{2}}{4 \theta^{\prime}} \int_{0}^{1} \theta^{2}(\xi, \lambda) d \xi
\end{aligned}
$$

Since $F_{\beta}(\lambda)=-2$, then the coefficient at the second integral equals zero. And the first addend differs from zero, since by assumption $\theta^{\prime} \neq 0$ and under the integral sign there is a nonnegative function not identically equal to zero.

Consequently, $F_{\beta}^{\prime}(\lambda) \neq 0$, that contradicts the assumption $F_{\beta}^{\prime}(\lambda)=0$. Hence, it follows that $\theta^{\prime}=0$.

Arguing as in the previous case, hence we find that the assumption $\varphi+\alpha \varphi^{\prime} \neq 0$ contradicts the condition $F_{\beta}^{\prime}(\lambda)=0$. Consequently, $\varphi+\alpha \varphi^{\prime}=0$.

In sequel, since $\theta^{\prime}=0$, then $\varphi^{\prime}+\theta=-2$ and $\theta \varphi^{\prime}=1$. Therefore $\theta+1 / \theta+2=0$ and $\theta=-1, \varphi^{\prime}=-1$.

At last, we prove that $F_{\beta}^{\prime \prime}(\lambda)>0$. 
Under the conditions $\theta=-1, \varphi^{\prime}=-1, \theta^{\prime}=0, \varphi=\alpha$, using the formula for derivatives $\partial \theta(\xi, \lambda) / \partial \lambda$ and $\partial \varphi(\xi, \lambda) / \partial \lambda$, we find that

$$
\frac{d^{2} F_{\beta}(\lambda)}{d \lambda^{2}}=2 \int_{0}^{1} d \xi \int_{0}^{\xi}[\varphi(\xi, \lambda) \theta(t, \lambda)-\varphi(t, \lambda) \theta(\xi, \lambda)]^{2} d t
$$

We find that $F_{\beta}^{\prime \prime}(\lambda)>0$.

The following theorem immediately follows from Lemmas 2.2-2.5.

Theorem 2.6. (1) Multiplicity of zeros of functions $\Phi_{ \pm}^{\alpha}(\lambda)$ and $\Phi_{ \pm}^{\beta}(\lambda)$ does not exceed two;

(2) at the points $\lambda$, where $\varphi^{\prime}+\alpha \varphi+\theta=2, F_{\alpha}^{\prime}(\lambda)=0$, the function $F_{\alpha}(\lambda)$ has a maximum;

(3) at the points $\lambda$, where $\varphi^{\prime}+\alpha \varphi+\theta=-2, F_{\alpha}^{\prime}(\lambda)=0$, the function $F_{\alpha}(\lambda)$ has a minimum;

(4) at the points $\lambda$, where $\varphi^{\prime}+\beta \theta^{\prime}+\theta=2, F_{\beta}^{\prime}(\lambda)=0$, the function $F_{\beta}(\lambda)$ has a maximum;

(5) at the points $\lambda$, where $\varphi^{\prime}+\beta \theta^{\prime}+\theta=-2, F_{\beta}^{\prime}(\lambda)=0$, the function $F_{\beta}(\lambda)$ has a minimum.

\section{Nature of the spectrum of the operator $L_{\alpha \beta}(q)$}

We denote by $H$ the Hilbert space $\ell_{2}(-\infty, \infty)$ and consider the $H$-valued functions $\hat{f}=$ $\left(\ldots, f_{n}(x), \ldots\right)(0 \leq x \leq 1)$, for which there exists the integral

$$
\int_{0}^{1}(\hat{f}, \hat{f})_{H} d x=\sum_{n=-\infty}^{\infty} \int_{0}^{1}\left|f_{n}(x)\right|^{2} d x
$$

Let us introduce the shift operator $T$ in $H$. For this, on the vector $U_{n}$ whose $n$th component equals unit, and the other components equal zero, we assume $T U_{n}=U_{n-1}, n=$ $0, \pm 1, \pm 2, \ldots$, and at the remaining vectors $x \in H$, the operator $T$ is determined by linearity. The operator $T$ is unitary, therefore, there exists an operator $T^{-1}=T^{*}$.

Partition the whole number axis into segments $\Delta_{k}=[k, k+1](k=0, \pm 1, \pm 2, \ldots)$. For $x \in \Delta_{n}$, put $x=n+t(0 \leq t \leq 1)$ and $y_{n}(t)=y(n+t)=y(x)$. From periodicity of the function $q(x)$, the assignment of the operator $L_{\alpha \beta}(q)$ in $L_{2}(-\infty, \infty)$ is equivalent in $H_{1}=$ $L_{2}([0,1], H)$ to the assignment of the expression

$$
\ell_{00}[\hat{y}(t)] \equiv-\hat{y}^{\prime \prime}(t)+q(t) E \hat{y}(t)
$$

with following boundary conditions. For $\delta$ point interactions,

$$
\left(\begin{array}{l}
\hat{y}^{\prime}(0) \\
\hat{y}^{\prime}(1)
\end{array}\right)=\alpha\left(\begin{array}{cc}
E & T^{-1} \\
T & E
\end{array}\right)\left(\begin{array}{c}
\hat{y}(0) \\
-\hat{y}(1)
\end{array}\right)
$$


but for $\delta^{\prime}$ point interactions,

$$
\left(\begin{array}{c}
\hat{y}(0) \\
-\hat{y}(1)
\end{array}\right)=\beta^{-1}\left(\begin{array}{cc}
E & T^{-1} \\
T & E
\end{array}\right)\left(\begin{array}{c}
\hat{y}^{\prime}(0) \\
\hat{y}^{\prime}(1)
\end{array}\right) .
$$

In this formula, $E$ is a unit operator in $H$. The following theorem is true.

TheOREM 3.1. Let $y_{s}(x, \lambda)$ th component of the vector function $\hat{y}(x, \lambda)$ be the solution of the equation

$$
\ell_{00}[\hat{y}]=\lambda \hat{y}+\hat{f} \quad\left(f \in H_{1}\right)
$$

with boundary conditions (3.3) and (3.4). Then

$$
\begin{aligned}
y_{s}(x, \lambda)= & \frac{\omega_{1}(x, \lambda)}{W(\lambda)} \sum_{p=s}^{\infty}\left[\rho_{2}(\lambda)\right]^{p-s} \int_{0}^{1} \omega_{2}(t, \lambda) f_{p}(t) d t \\
& +\frac{\omega_{2}(x, \lambda)}{W(\lambda)} \sum_{p=-\infty}^{s}\left[\rho_{1}(\lambda)\right]^{p-s} \int_{0}^{1} \omega_{1}(t, \lambda) f_{p}(t) d t \\
& -\frac{1}{W(\lambda)}\left[\omega_{1}(x, \lambda) \int_{0}^{x} \omega_{2}(t, \lambda) f_{s}(t) d t+\omega_{2}(x, \lambda) \int_{x}^{1} \omega_{1}(t, \lambda) f_{s}(t) d t\right]
\end{aligned}
$$

where $\omega_{j}(x, \lambda)=\theta(x, \lambda)+m_{j}(\lambda) \varphi(x, \lambda), W(\lambda)=m_{1}(\lambda)-m_{2}(\lambda), m_{i}(\lambda)(i=1,2)$ are the solutions of the equations

$$
\begin{aligned}
& \varphi m^{2}+\left(\theta-\alpha \varphi-\varphi^{\prime}\right) m-\left[\theta+\alpha \theta^{\prime}\right]=0 \quad \text { for } s \leq m, \\
& \theta^{\prime} m^{2}+\left(\varphi-\theta-\beta \theta^{\prime}\right) m-\left[\varphi+\beta \varphi^{\prime}\right]=0 \text { for } s>m \text {, } \\
& \rho_{j}(\lambda)=\left\{\begin{array}{lll}
\varphi^{\prime}+\alpha \varphi-m_{j}(\lambda) \varphi & (j=1,2) & \text { if } s \leq m, \\
\theta+\beta \theta^{\prime}-m_{j}(\lambda) \theta^{\prime} & (j=1,2) & \text { if } s>m .
\end{array}\right.
\end{aligned}
$$

By formula (3.6), the spectrum of the operator $L_{\alpha \beta}(q)$ coincides with singularities of the analytic function $W^{-1}(\lambda)=\left(m_{1}(\lambda)-m_{2}(\lambda)\right)^{-1}$. Since

$$
W(\lambda)= \begin{cases}{[\varphi(\lambda)]^{-1} \sqrt{\Phi_{+}^{\alpha}(\lambda) \cdot \Phi_{-}^{\alpha}(\lambda)}} & \text { if } s \leq m, \\ \cdot\left[\theta^{\prime}(\lambda)\right]^{-1} \sqrt{\Phi_{+}^{\beta}(\lambda) \cdot \Phi_{-}^{\beta}(\lambda)} & \text { if } s>m,\end{cases}
$$

then it follows from the results of Section 2 that the function $W^{-1}(\lambda)$ has branching points of the second order at the points $k_{n}$ and $\mu_{n}$, if they are simple zeros of the functions $\Phi_{ \pm}^{\alpha}(\lambda)$ and $\Phi_{ \pm}^{\beta}(\lambda)$. At the multiple zeros of functions, $\Phi_{ \pm}^{\alpha}(\lambda) \varphi(\lambda)\left(\sqrt{\Phi_{+}^{\alpha}(\lambda) . \Phi_{-}^{\alpha}(\lambda)}\right)^{-1}$ and $\Phi_{ \pm}^{\beta}(\lambda) \theta^{\prime}(\lambda)\left(\sqrt{\Phi_{+}^{\beta}(\lambda) . \Phi_{-}^{\beta}(\lambda)}\right)^{-1}$ are regular, since at these points $\varphi(\lambda)=0$ and $\theta^{\prime}(\lambda)=$ 0 , respectively. Consequently, the spectrum of the operator $L_{\alpha \beta}(q)$ coincides with segments of the real axis $\left[k_{2 j}, \mu_{2 j}\right],\left[\mu_{2 j+1}, k_{2 j+1}\right], j=0, \pm 1, \pm 2, \ldots$ Intervals of the real axis $\left(\mu_{j}, \mu_{j+1}\right),\left(k_{j}, k_{j+1}\right), j=0, \pm 1, \pm 2, \ldots$, are the gaps in the spectrum. 
Assume that $q(x)=0$. Then $\lambda<0$ is eigenvalues of the operator $L_{\alpha \beta}(q)$ if and only if $z=i \sqrt{|\lambda|}$ is the solution of the equation

$$
\left(2 z-\beta-\alpha z^{2}+\alpha \beta z\right) e^{-2 z}+\left(2 z+\beta+\alpha z^{2}+\alpha \beta z\right) e^{2 z}=4 z .
$$

The number of solutions of (3.10) can determinate with graphic. Then it is easily shown that following expressions are true:

(1) if $\alpha>0, \beta>0$, then the operator $L_{\alpha \beta}(0)$ has no eigenvalue;

(2) if $\alpha \beta<0$, then the operator $L_{\alpha \beta}(0)$ has only one eigenvalue;

(3) if $\alpha<0, \beta<0$, then the operator $L_{\alpha \beta}(0)$ has only two eigenvalues.

As is known for Sturm-Liouville operator with periodic continuous potential $q(x)$, the length of gaps in the spectrum, if they are infinite number, tends to zero for unlimited increase of numbers of gaps (see [8]). In our case, the following theorem is valid.

THEOREM 3.2. The number of gaps in the spectrum of the operator $L_{\alpha \beta}(q)$ is infinite, and for unlimited increase of numbers of gaps, the lengths of gaps tend to nonzero constant in some place and to infinity in another place.

Proof. Estimate asymptotic behavior of numbers $k_{j}$ and $\mu_{j}$ for large values of $j$. Conduct this estimation for $k_{j}$ ( $\mu_{j}$ estimated similarly) in detail.

If we apply arguments stated in [8, Part 2, pages 353-354] to the functions $\Phi_{-}^{\alpha}(\lambda)=$ $\varphi^{\prime}+\alpha \varphi+\theta-2$ and $\Phi_{-}^{\beta}(\lambda)=\varphi^{\prime}+\beta \theta^{\prime}+\theta-2$, then we arrive to the following result:

$$
k_{n}^{2}\left(k_{2 n-1}^{2}\right)= \begin{cases}4 \pi^{2} n^{2}+\frac{a_{0}+2}{2} \pm \frac{1}{2} \sqrt{4 \alpha^{2}+a_{2 n}^{2}+b_{2 n}^{2}+4 a_{2 n}}+O\left(\frac{1}{n}\right) & \text { if } 2 n \leq m, \\ 4 \pi^{2} n^{2}+\frac{a_{0}}{2} \pm \frac{1}{2} \sqrt{a_{2 n}^{2}+b_{2 n}^{2}+16 \beta^{2} \pi^{2} n^{2}-8 \alpha n b_{2 n}}+O\left(\frac{1}{n}\right), & \text { if } 2 n>m,\end{cases}
$$

and since $\int_{0}^{1} q(x) d x<\infty$, then

$$
k_{2 n}^{2}-k_{2 n-1}^{2}= \begin{cases}2|\alpha|+O(1) & \text { if } 2 n \leq m, \\ 4|\beta| \pi n+O(1) & \text { if } 2 n>m,\end{cases}
$$

where

$$
a_{n}=2 \int_{0}^{1} q(y) \cos 2 \pi n y d y, \quad b_{n}=2 \int_{0}^{1} q(y) \sin 2 \pi n y d y
$$

\section{Acknowledgment}

The author is greatly indebted to Professor M.G. Gasymov for his constructive suggestions. 


\section{References}

[1] F. A. Berezin and L. D. Faddeev, "Remark on the Schrödinger's equation with singular potential," Doklady Akademii Nauk SSSR, vol. 137, pp. 1011-1014, 1961 (Russia).

[2] M. A. Naĭmark, Linear Differential Operators. Part II: Linear Differential Operators in Hilbert Space, Frederick Ungar Publishing, New York, NY, USA, 1968.

[3] N. Dunford and J. T. Schwartz, Linear Operators, Part II, Spectral Theory, John Wiley \& Sons, New York, NY, USA, 1964.

[4] F. S. Roffe-Beketov, "Self-adjoint extensions of differential operators in the space of vectorfunctions," Doklady Akademii Nauk SSSR, vol. 184, no. 5, pp. 1034-1037, 1969 (Russian).

[5] F. Gesztesy and H. Holden, "A new class of solvable models in quantum mechanics describing point interactions on the line," Journal of Physics A, vol. 20, no. 15, pp. 5157-5177, 1987.

[6] S. Albererio, F. Gesztezy, R. Høegh-Krohn, and H. Holden, Solvable Models in Quantum Mechanics, AMS Chelsea, Providence, RI, USA, 2nd edition, 1988.

[7] V. A. Mikhălets, "On the Schrödinger operator with point $\delta$ '-interactions," Doklady Akademii Nauk SSSR, vol. 348, no. 6, pp. 727-730, 1996 (Russian).

[8] E. C. Titchmarsh, Eigenfunction Expansions Associated with Second-Order Differential Equations. Part I, Clarendon Press, Oxford, UK, 2nd edition, 1962.

[9] M. M. Gehtman and I. V. Stankevich, "A generalized Kronig-Penney problem," Akademija Nauk SSSR, vol. 11, no. 1, pp. 61-62, 1977 (Russian).

[10] P. Kurasov and J. Larson, "Spectral asymptotics for Schrödinger operators with periodic point interactions," Journal of Mathematical Analysis and Applications, vol. 266, no. 1, pp. 127-148, 2002.

Mehmet Sahin: Department of Mathematics, Faculty of Arts and Sciences, Gaziantep University, 27310 Gaziantep, Turkey

Email address: mesahin@gantep.edu.tr

Manaf Dzh. Manafov: Department of Mathematics, Faculty of Arts and Sciences,

Gaziantep University, 27310 Gaziantep, Turkey

Email address: manafov@gantep.edu.tr 


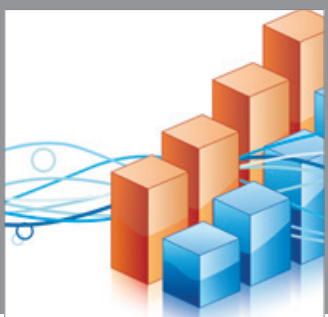

Advances in

Operations Research

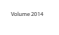

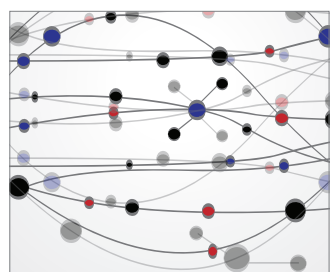

\section{The Scientific} World Journal
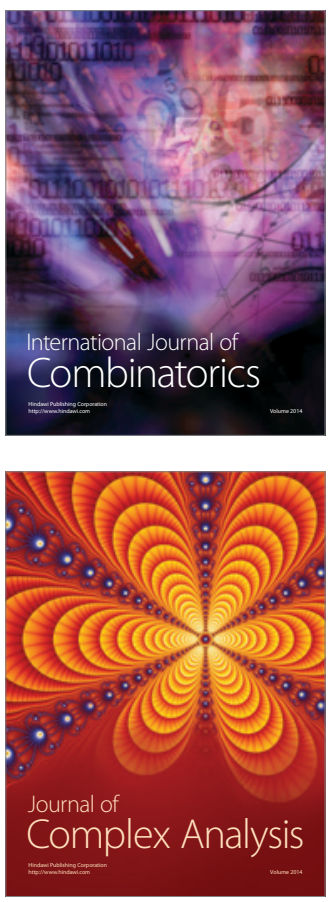

International Journal of

Mathematics and

Mathematical

Sciences
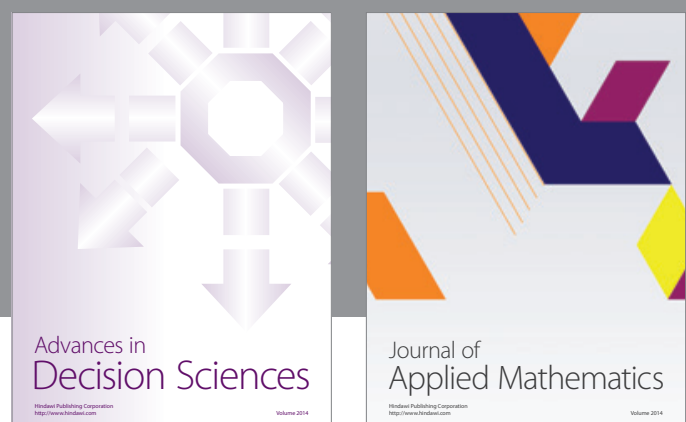

Journal of

Applied Mathematics
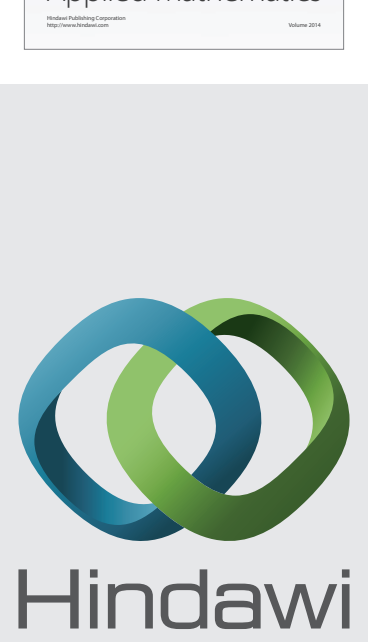

Submit your manuscripts at http://www.hindawi.com
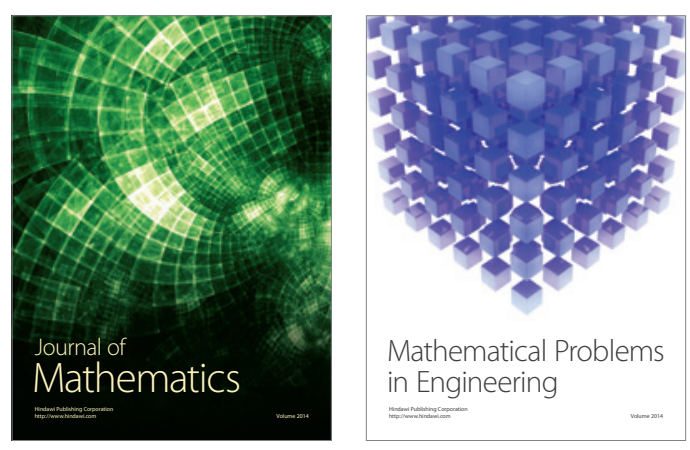

Mathematical Problems in Engineering
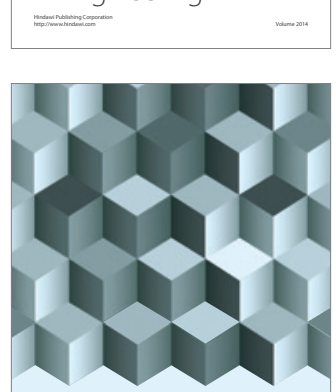

Journal of

Function Spaces
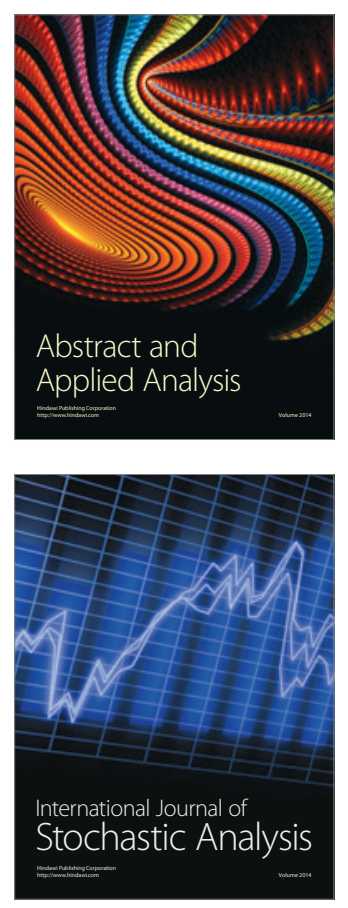

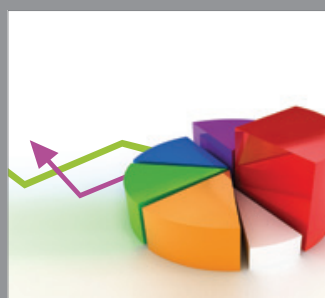

ournal of

Probability and Statistics

Promensencen
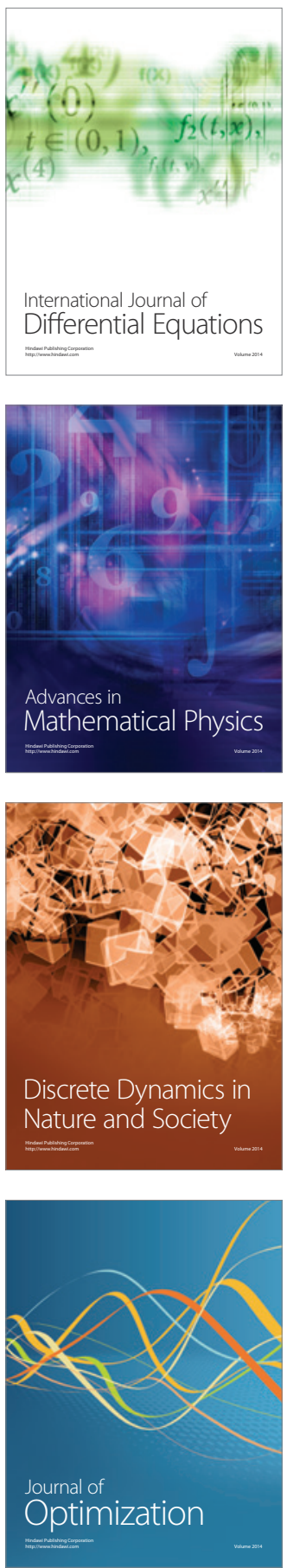